\title{
Outcomes of re-irradiation for brain recurrence after prophylactic or therapeutic whole-brain irradiation for small cell lung Cancer: a retrospective analysis
}

Ryoko Suzuki ${ }^{1}$, Xiong Wei ${ }^{1}$, Pamela K. Allen ${ }^{1}$, James W. Welsh ${ }^{1}$, James D. Cox ${ }^{1}$, Ritsuko Komaki ${ }^{1}$ and Steven H. Lin ${ }^{1,2^{*}}$

\begin{abstract}
Background: Small cell lung cancer (SCLC) can recur in the brain after whole-brain irradiation (WBI). We documented outcomes after treatment of such recurrences and sought predictors of local control and overall survival (OS).

Materials and methods: Eighty-five patients with SCLC and brain recurrence after prophylactic or therapeutic WBI in 1998-2015 were identified and data were extracted from the medical records. Survival was estimated with the Kaplan-Meier method, and univariate and multivariate Cox proportional hazards modeling was used to identify factors associated with OS or further brain progression.

Results: Brain recurrence was treated by stereotactic radiosurgery (SRS) in 33 patients (39\%), repeat WBI in 14 (16\%), chemotherapy-only in 16 (19\%), and observation in 22 (26\%). Median OS time after brain recurrence (OSrec) was 4.3 months for all patients; 6-month OSrec rates were 58\% after SRS, 21\% after repeat WBI, 50\% after chemotherapy-only, and 5\% after observation $(P<0.001$ ). Inferior OSrec was associated with poor performance status (ECOG score $\geq 3$ ) and uncontrolled extracranial disease. Superior OSrec was associated with receipt of $\geq 4$ chemotherapy cycles before brain recurrence and receipt of chemotherapy, SRS, or repeat WBI afterward. Receipt of chemotherapy after brain recurrence correlated with brain progression.
\end{abstract}

Conclusions: Some patients with brain recurrence after WBI for SCLC can survive for extended periods with appropriate intervention, especially those with adequate performance status or controlled extracranial disease.

Keywords: CNS control, WBRT, Stereotactic radiosurgery, Survival

\section{Background}

Whole-brain irradiation (WBI) is an important component of treatment for small cell lung cancer (SCLC), whether given prophylactically or to treat known brain metastases. Because SCLC has a high propensity to seed in the brain, disease recurrence in the brain after WBI is not uncommon despite the radiosensitivity of the disease $[1,2]$. Treatment strategies for brain recurrence after initial therapy for SCLC are limited but include surgical

\footnotetext{
* Correspondence: SHLin@mdanderson.org

'Department of Radiation Oncology, Unit 97, The University of Texas MD Anderson Cancer Center, 1515 Holcombe Blvd, Houston, TX 77030-4009, USA

${ }^{2}$ Present address: Department of Radiation Oncology, Tokyo Medical and Dental University, 1 Chome-5-45 Yushima, Bunkyō, Tokyo 113-8510, Japan
}

resection, stereotactic radiosurgery (SRS), repeat WBI, and chemotherapy.

In one autopsy series, $65 \%$ of patients had evidence of brain metastases at autopsy, with the probability of brain metastases increasing with survival time to $80 \%$ after 2 years [3]. Extended survival times from the use of modern radiotherapy techniques $[4,5]$ and the enhanced ability to detect small asymptomatic brain metastases [6] collectively increase the likelihood that more patients will manifest brain recurrence after initial WBI therapy for SCLC [6].

Repeat WBI for brain metastases from a variety of tumor types including lung cancer has been shown to be effective for symptom palliation [7-11]. A benefit from

(C) The Author(s). 2018 Open Access This article is distributed under the terms of the Creative Commons Attribution 4.0 International License (http://creativecommons.org/licenses/by/4.0/), which permits unrestricted use, distribution, and 
re-irradiating brain recurrences has also been found for patients with SCLC, although the median survival time after re-irradiation remains relatively short at 2 to 6 months $[2,12,13]$. In one study, patients with SCLC who developed brain metastases after prophylactic cranial irradiation (PCI) had effective symptom palliation from either repeat WBI or SRS [12]. Another series of patients with SCLC and brain relapse after WBI confirmed the feasibility of SRS as a salvage option [13, 14].

Nevertheless, information on how best to treat SCLC that has recurred in the brain after WBI is still sparse. Considering the high prevalence and depressing prognosis associated with brain recurrence, identifying reliable prognostic factors would be helpful for choosing among the various available treatment strategies.

In this single-institution retrospective study, we evaluated patients with SCLC who developed brain recurrence after WBI, with the goals of assessing outcomes and identifying prognostic factors that could influence survival and brain control.

\section{Methods}

After approval of this study by the appropriate institutional review board, we identified 85 patients with SCLC and brain recurrence after WBI, given either with therapeutic or prophylactic intent, from 1998 through 2015 at a single tertiary cancer center. Management strategies for those brain recurrences (SRS, repeat WBI, chemotherapy, or observation) were extracted from the patients' medical records, as was the following demographic and clinical information: factors at the initial diagnosis [sex, race, initial disease stage (limited vs. extensive), the presence and number of brain metastases at initial diagnosis ( $\leq 4 \mathrm{vs}$. $>4$ ), intent of the first WBI (prophylactic vs. therapeutic), number of chemotherapy cycles before brain recurrences $(<4$ vs. $\geq 4)$, and use and dose of thoracic radiation therapy ( $<45$ Gy vs. $\geq 45 \mathrm{~Gy}$ )]; factors at the brain recurrence [age, ECOG performance status (PS), symptoms and number of brain lesions $(\leq 4$ vs. $>4)$, the presence and status of extracranial disease (controlled vs. uncontrolled), and time from the initial WBI to the brain recurrence $(<6$ months vs. $\geq 6$ months)]; and factors after brain recurrence [treatment strategy (SRS, repeat WBI, chemotherapy only, or observation)]. Patients were typically given steroids, especially if they had symptoms, immediately upon diagnosis of brain recurrence. Patients with brain recurrence managed with observation were followed with best supportive care, including drugs such as steroids for symptom control.

The primary endpoint of this study was overall survival after brain recurrence (OSrec), which was defined as the time from the date of brain recurrence to the date of death. Local control in the brain after brain recurrence (BCrec), evaluated radiologically or clinically, was defined as the time from the date of brain recurrence to the date of detection of further brain recurrence. Patients lost to follow-up were censored at the date of the last follow-up. Progression was defined as lesions that persisted after SRS, repeat WBI, or chemotherapy or, in the observation group, as brain recurrence. The Kaplan-Meier method was used to assess survival and progression probabilities, and log-rank tests were used to compare Kaplan-Meier estimates of event rates between potential predictors. Univariate and multivariate Cox proportional hazards models were used to assess associations between factors and survival or further disease progression in the brain, and those factors with a $P$ value $<0.1$ were used to construct the final multivariate Cox model. All analyses were two-sided, and significance was set at $P<0.05$. Statistical analyses were done with SPSS version 24 (SPSS, Chicago, IL, USA).

\section{Results}

Patient characteristics, stratified by treatment for brain recurrence, are summarized in Table 1 . Among all 85 patients with brain recurrence after WBI, 10 (12\%) had poor PS (i.e., ECOG 3-4); 24 (28\%) had had limited SCLC; and 33 (39\%) had received WBI for prophylaxis, with the other $52(61 \%)$ receiving WBI with therapeutic intent. All 85 patients had extracranial disease at brain recurrence, which was controlled in 29 (34\%). The median interval between the first WBI and the brain recurrence was 6.0 months (range, 0.8-89.5 months).

Brain recurrence was managed with SRS in 33 patients (39\%), with repeat WBI in 14 (16\%), with chemotherapy only in 16 (19\%), and with observation (and best supportive care) in the remaining 22 patients (26\%). Two of the patients in the SRS group had received SRS after craniotomy owing to the size of the lesion. One patient in the repeat WBI group had local fractionated radiotherapy rather than WBI. Of the 33 patients in the SRS group, $27(82 \%)$ had $\leq 4$ brain lesions at recurrence; by contrast, 9 patients (64\%) in the repeat WBI group and 13 patients (59\%) in the observation group had $>4$ lesions. More than a third of patients in the SRS group also received additional chemotherapy, but only one patient in the repeat WBI group and no one in the observation group received further chemotherapy.

Nineteen patients in the SRS group (58\%) had further brain failure, and 5 of those patients received a second SRS; 2 patients who received a second SRS had a third recurrence, which was treated with salvage WBI; and 1 was treated with salvage WBI. Thirteen patients in the chemotherapy-only group (81\%) had further brain failure; 1 was treated with SRS, 1 was treated with SRS and had a second recurrence also treated with SRS, 2 were treated with SRS and had a second recurrence treated with salvage WBI, and 3 were treated with salvage WBI. 
Table 1 Patient characteristics by treatment received for brain recurrence

\begin{tabular}{|c|c|c|c|c|c|}
\hline & $\begin{array}{l}\text { All Patients } \\
(n=85)\end{array}$ & $\begin{array}{l}\text { Group } 1 \text { (SRS) } \\
(n=33)^{\mathrm{a}}\end{array}$ & $\begin{array}{l}\text { Group 2 (WBI) } \\
(n=14)^{b}\end{array}$ & $\begin{array}{l}\text { Group } 3 \text { (Chemo only) } \\
(n=16)\end{array}$ & $\begin{array}{l}\text { Group } 4 \text { (Observation) } \\
(n=22)\end{array}$ \\
\hline \multicolumn{6}{|c|}{ Age at brain recurrence, years } \\
\hline$<63$ & 42 & 16 & 6 & 10 & 10 \\
\hline$\geq 63$ & 43 & 17 & 8 & 6 & 12 \\
\hline \multicolumn{6}{|c|}{ ECOG performance status score at brain recurrence } \\
\hline $0-2$ & 75 & 32 & 11 & 15 & 17 \\
\hline$\geq 3$ & 10 & 1 & 3 & 1 & 5 \\
\hline \multicolumn{6}{|l|}{ Sex } \\
\hline Male & 33 & 12 & 6 & 6 & 9 \\
\hline Female & 52 & 21 & 8 & 10 & 13 \\
\hline \multicolumn{6}{|l|}{ Ethnicity } \\
\hline White & 69 & 23 & 11 & 15 & 20 \\
\hline Non-white & 16 & 10 & 3 & 1 & 2 \\
\hline \multicolumn{6}{|c|}{ Initial disease stage } \\
\hline Limited & 24 & 12 & 3 & 2 & 7 \\
\hline Extensive & 61 & 21 & 11 & 14 & 15 \\
\hline \multicolumn{6}{|c|}{ Brain metastases at initial diagnosis } \\
\hline No & 52 & 23 & 7 & 8 & 14 \\
\hline Yes & 33 & 10 & 7 & 8 & 8 \\
\hline
\end{tabular}

No. of brain lesions at initial diagnosis

\begin{tabular}{|c|c|c|c|c|}
\hline$\leq 4$ & 72 & 30 & 10 & 13 \\
\hline$>4$ & 13 & 3 & 4 & 3 \\
\hline
\end{tabular}

Intent of first brain irradiation

$\begin{array}{lll}\text { Prophylactic } & 33 & 14 \\ \text { Therapeutic } & 52 & 19\end{array}$

5

5

$\geq 4$ chemo cycles before brain recurrence

$\begin{array}{lll}\text { No } & 5 & 1 \\ \text { Yes } & 80 & 32\end{array}$

Yes

80

32

9

Thoracic radiation therapy dose, Gy

$\begin{array}{llllll}<45 & 28 & 6 & 7 & 7 & 8 \\ \geq 45 & 57 & 27 & 7 & 9 & 14\end{array}$

No. of brain lesions at brain recurrence

$\begin{array}{lll}\leq 4 & 46 & 27 \\ >4 & 39 & 6\end{array}$

Symptoms from brain lesions at brain recurrence

$\begin{array}{lll}\text { No } & 49 & 25 \\ \text { Yes } & 36 & 8\end{array}$

Extracranial disease at brain recurrence

$\begin{array}{lll}\text { Controlled } & 29 & 15 \\ \text { Uncontrolled } & 56 & 18\end{array}$

Chemotherapy after brain recurrence

$\begin{array}{lll}\text { No } & 55 & 20 \\ \text { Yes } & 30 & 13\end{array}$

20
13

25


Table 1 Patient characteristics by treatment received for brain recurrence (Continued)

\begin{tabular}{llllll}
\hline & $\begin{array}{l}\text { All Patients } \\
(n=85)\end{array}$ & $\begin{array}{l}\text { Group 1 (SRS) } \\
(n=33)^{a}\end{array}$ & $\begin{array}{l}\text { Group 2 (WBI) } \\
(n=14)^{b}\end{array}$ & $\begin{array}{l}\text { Group 3 (Chemo only) } \\
(n=16)\end{array}$ & $\begin{array}{l}\text { Group 4 (Observation) } \\
(n=22)\end{array}$ \\
\hline Time from the end of first brain irradiation to brain recurrence, mo & & & 16 \\
$<6$ & 44 & 13 & 7 & 8 & 6 \\
$\geq 6$ & 41 & 20 & 7 & 8 & 6 \\
\hline
\end{tabular}

${ }^{a}$ Two patients received SRS with a Gamma knife after craniotomy

${ }^{b}$ One patient received local (not whole-brain) radiation to tumor site using conventional radiotherapy

Abbreviations: SRS stereotactic radiosurgery, WBI whole-brain irradiation, ECOG Eastern Cooperative Oncology Group

Ten patients in the repeat-WBI group (71\%) had further brain failure, of whom 1 had a craniotomy and the other 9 received best supportive care.

The median total dose of the first course of WBI was 30 Gy (range, 20-36 Gy), given in a median 3 Gy per fraction (range, 2-3Gy). Most patients given repeat WBI received $20 \mathrm{~Gy}$ in ten 2-Gy fractions. For patients who received SRS, the prescribed dose was 12-20 Gy per lesion depending on their size and location.

The median OSrec time (i.e., survival after brain recurrence) was 4.3 months (95\% confidence interval [CI] 2.7-5.9) for the whole group. Median OSrec time for those with ECOG PS 0-2 at recurrence was 5.0 months (95\% CI 3.4-6.7) compared with 1.2 months (95\% CI $0.1-2.2)$ for those with PS $\geq 3 \quad(P<0.001)$ (Fig. 1a). Median OSrec time for those with extracranial disease control at brain recurrence was 9.1 months (95\% CI 1.916.4) compared with 3.2 months (95\% CI 2.3-4.1) for those with uncontrolled extracranial disease $(P<0.001)$ (Fig. 1b). Rates of OSrec at 6 months were different according to type of treatment for the recurrence: 58\% for the SRS group, 21\% for the repeat WBI group, 50\% for the chemotherapy-only group, and $5 \%$ for the observation group $(P<0.001)$ (Fig. 1c).

Further brain progression during follow-up was noted in 59 of the 85 patients. The median BCrec interval (i.e., brain control after brain recurrence) was 3.6 months (95\% CI 2.2-5.1) for the entire group.

As for factors influencing OSrec, univariate analysis showed that worse OSrec was associated with poor PS $(\geq 3)$ at recurrence, the presence of symptomatic brain lesions, and the presence of uncontrolled extracranial disease; better OSrec was associated with receipt of $\geq 4$ chemotherapy cycles before brain recurrence, receipt of chemotherapy after brain recurrence, receipt of repeat WBI or SRS for brain recurrence, and an interval of $\geq 6$ months from the end of the first WBI to the brain recurrence. Univariate analysis for factors associated with $\mathrm{BCrec}$ showed that worse BCrec was associated with poor PS $(\geq 3)$, $>4$ brain lesions at recurrence, symptomatic brain lesions, and uncontrolled extracranial disease; better $\mathrm{BCrec}$ was associated with receipt of repeat WBI or SRS for the brain recurrence. OSrec and $\mathrm{BCrec}$ did not differ for patients with limitedversus extensive-stage disease at initial diagnosis (Table 2).
In multivariate analysis, worse OSrec was associated with poor PS $(\geq 3)$ at brain recurrence (hazard ratio [HR] 3.039, 95\% CI 1.374-6.721, $P=0.006$ ) and uncontrolled extracranial disease (HR 3.059, 95\% CI 1.665-5.622, $P<$ 0.001 ). Improved OSrec was associated with receipt of $\geq 4$ chemotherapy cycles before brain recurrence (HR $0.351,95 \%$ CI $0.126-0.975, P=0.045)$; receipt of chemotherapy for brain recurrence (HR 0.174, 95\% CI 0.094$0.324, P<0.001)$; and receipt of repeat brain irradiation (with WBI or Gamma-knife) for brain recurrence (HR 0.367; 95\% CI 0.205-0.656; $P<0.001)$. As for BCrec, receipt of chemotherapy after brain recurrence was borderline significant in univariate analysis for $\mathrm{BCrec}(P=$ 0.085 ) but became significant in multivariate analysis after adjustment for other variables that were significant at $P<0.1$ in univariate analysis (HR $0.457,95 \% \mathrm{CI}$ $0.250-0.832, \quad P=0.01$ ), indicating that receipt of chemotherapy after brain recurrence may be associated with better BCrec. Uncontrolled extracranial disease and receipt of repeat brain irradiation (with WBI or Gamma-knife) for brain recurrence were marginally significant in multivariate analysis of $\mathrm{BCrec}(P=0.055$ and $P=0.050)$. No other factors were found to be associated with $\mathrm{BCrec}$ in multivariate analysis (Table 3 ).

Ten of the 85 patients in our study lived for more than 1 year after the first brain recurrence (Table 4). Notably, 8 of those 10 patients did not have liver or bone involvement at initial diagnosis. Moreover, Patients 5 and 7, who received temozolomide (without re-irradiation) for the initial recurrence later underwent SRS for a subsequent brain recurrence. Therefore all 10 of these patients had received SRS, whether for the initial or subsequent brain recurrence.

\section{Discussion}

Available options for patients with disease recurrence in the brain after WBI for SCLC are limited, but our findings suggest that some patients can expect better survival when those recurrences are treated with therapeutic intent by using SRS, repeat WBI, or chemotherapy, especially patients with adequate PS or controlled extracranial disease.

Despite the radiosensitivity of SCLC, brain recurrence after WBI is not uncommon [1,2]. In one meta-analysis 


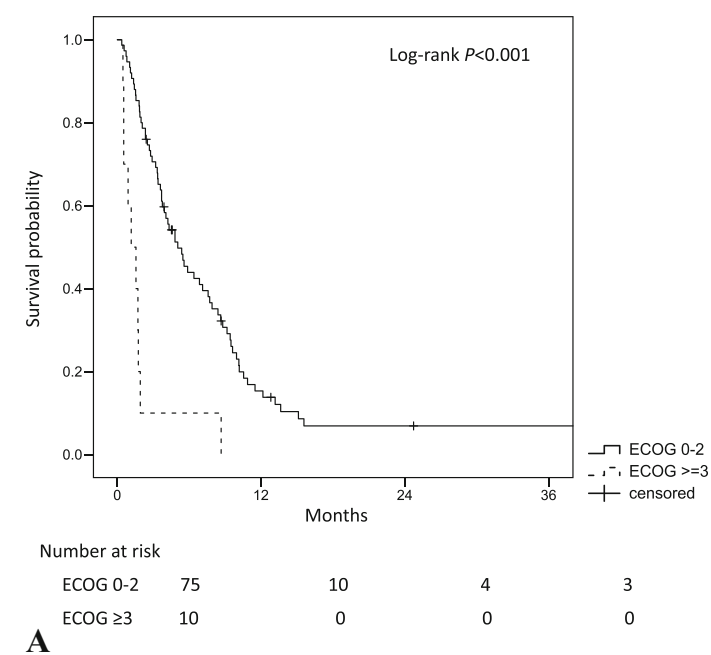

A

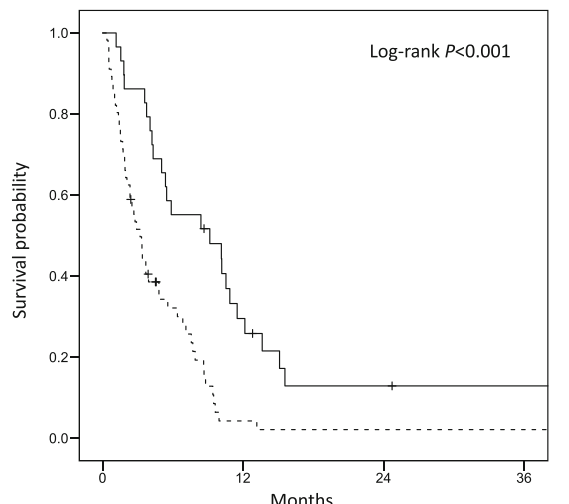

$\neg$ Controlled $-\because$ Uncontrolled
+

Number at risk

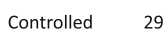

Uncontrolled 56

B

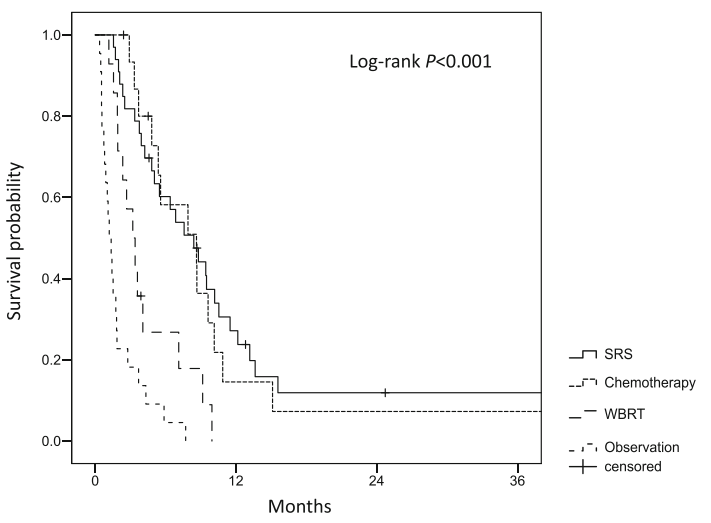

Number at risk

SRS 33

Chemotherapy 16

Repeat WBI 14

C
Observation 22

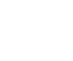

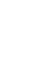


Table 2 Univariate analysis of factors affecting survival and further brain control after recurrence in the brain after therapeutic or prophylactic irradiation

\begin{tabular}{lllll}
\hline Characteristics & Survival after Brain Failure & & Brain Control after Brain Failure \\
\cline { 2 - 3 } & Hazard Ratio $(95 \% \mathrm{Cl})$ & $P$ Value & Hazard Ratio $(95 \% \mathrm{Cl})$ & \\
\hline $\begin{array}{l}\text { Age at brain recurrence, years } \\
<63\end{array}$ & 1.00 & 0.757 & 1.00 \\
$\geq 63$ & $0.931(0.590-1.468)$ & & $0.881(0.526-1.478)$
\end{tabular}

ECOG performance status score at brain recurrence

$$
\begin{array}{ll}
0-2 & 1.00 \\
\geq 3 & 4.66(2.310-9.403)
\end{array}
$$

Sex

$\begin{array}{ll}\text { Male } & 1.00 \\ \text { Female } & 0.985(0.615-1.580)\end{array}$

Ethnicity

$$
\text { White }
$$

1.00

Non-white

$0.762(0.418-1.386)$

Initial disease stage

$\begin{array}{ll}\text { Limited } & 1.00 \\ \text { Extensive } & 1.015(0.605-1.703)\end{array}$

Brain metastases at initial diagnosis

$$
\begin{array}{ll}
\text { No } & 1.00 \\
\text { Yes } & 1.112(0.698-1.771)
\end{array}
$$

No. of brain lesions at initial diagnosis

$$
\leq 4
$$$$
1.00
$$$$
>4
$$$$
0.963(0.518-1.793)
$$

Intent of first brain irradiation

$\begin{array}{ll}\text { Prophylactic } & 1.00 \\ \text { Therapeutic } & 0.973(0.606-1.564)\end{array}$

$\geq 4$ chemo cycles before brain recurrence

$$
\begin{array}{ll}
\text { No } & 1.00 \\
\text { Yes } & 0.236(0.091-0.614)
\end{array}
$$

Thoracic radiation therapy dose, Gy

$\begin{array}{ll}<45 & 1.00 \\ \geq 45 & 0.844(0.524-1.361)\end{array}$

No. of brain lesions at brain recurrence

$$
\leq 4
$$$$
1.00
$$$$
>4
$$

$1.552(0.981-2.454)$

Symptoms from brain metastases at brain recurrence

$$
\begin{array}{ll}
\text { No } & 1.00 \\
\text { Yes } & 2.041(1.265-3.294)
\end{array}
$$

Extracranial disease at brain recurrence

$\begin{array}{ll}\text { Controlled } & 1.00 \\ \text { Uncontrolled } & 2.683(1.603-4.490)\end{array}$

Chemotherapy after brain recurrence

$\begin{array}{ll}\text { No } & 1.00 \\ \text { Yes } & 0.406(0.248-0.663)\end{array}$

$<0.001 \quad 1.00$

0.007

$2.776(1.318-5.849)$

0.951

1.00

$1.285(0.751-2.199)$

0.373

1.00

0.801

$0.921(0.485-1.748)$

0.955

1.00

$1.601(0.872-2.940)$

0.656

1.00

0.519

$1.19(0.701-2.020)$

0.906

1.00

0.624

$1.179(0.610-2.278)$

0.910

1.00

0.853

$1.051(0.621-1.778)$

0.003

1.00

0.882

$0.897(0.215-3.752)$

0.487

1.00

0.774

$0.923(0.532-1.600)$

0.060

1.00

0.033

1.792 (1.049-3.063)

0.003

1.00

0.043

$1.718(1.018-2.902)$

$<0.001$

1.00

0.012

2.084 (1.177-3.689)

$<0.001$

1.00

0.085

$0.62(0.360-1.069)$ 
Table 2 Univariate analysis of factors affecting survival and further brain control after recurrence in the brain after therapeutic or prophylactic irradiation (Continued)

\begin{tabular}{|c|c|c|c|c|}
\hline \multirow[t]{2}{*}{ Characteristics } & \multicolumn{2}{|c|}{ Survival after Brain Failure } & \multicolumn{2}{|c|}{ Brain Control after Brain Failure } \\
\hline & Hazard Ratio $(95 \% \mathrm{Cl})$ & $P$ Value & Hazard Ratio $(95 \% \mathrm{Cl})$ & $P$ Value \\
\hline \multicolumn{5}{|c|}{ WBI or G-knife for brain recurrence } \\
\hline No & 1.00 & 0.018 & 1.00 & 0.009 \\
\hline Yes & $0.576(0.365-0.908)$ & & $0.494(0.290-0.842)$ & \\
\hline \multicolumn{5}{|c|}{ Time from the end of first brain irradiation to brain recurrence, mo } \\
\hline$<6$ & 1.00 & 0.034 & 1.00 & 0.332 \\
\hline$\geq 6$ & $0.608(0.384-0.963)$ & & $0.77(0.460-1.291)$ & \\
\hline
\end{tabular}

Abbreviations: ECOG Eastern Cooperative Oncology Group, WBI whole-brain irradiation, G-knife Gamma knife therapy

after re-irradiation, and the median survival times were 3 months after repeat-WBI and 5 months after SRS. That study further showed that patients with higher KPS $(\geq 50)$ had significantly improved survival after re-irradiation, a finding that is comparable to ours [12]. The importance of PS in survival was also confirmed in a study that used salvage SRS after WBI; other factors found to be significant were tumor volume and the interval between SCLC diagnosis and the appearance of brain metastases [14]. Another retrospective study assessing the efficacy of salvage SRS after WBI for SCLC showed that although the median OS time for all patients was 5.9 months, $24 \%$ of patients survived for at least 12 months, and patients with no evidence of extracranial disease had

Table 3 Multivariate analysis of factors affecting survival and further brain control after recurrence in the brain after therapeutic or prophylactic irradiation

\begin{tabular}{|c|c|c|c|c|}
\hline \multirow[t]{2}{*}{ Characteristics } & \multicolumn{2}{|c|}{ Survival after Brain Failure } & \multicolumn{2}{|c|}{ Brain Control after Brain Failure } \\
\hline & Hazard Ratio $(95 \% \mathrm{Cl})$ & $P$ Value & Hazard Ratio (95\% Cl) & $P$ Value \\
\hline \multicolumn{5}{|c|}{ ECOG performance status score at brain recurrence } \\
\hline $0-2$ & 1.00 & 0.006 & 1.00 & 0.103 \\
\hline$\geq 3$ & $3.039(1.374-6.721)$ & & $1.955(0.874-4.372)$ & \\
\hline \multicolumn{5}{|c|}{$\geq 4$ chemo cycles before brain recurrence } \\
\hline No & 1.00 & 0.045 & - & - \\
\hline Yes & $0.351(0.126-0.975)$ & & & - \\
\hline \multicolumn{5}{|c|}{ No. of brain lesions at brain recurrence } \\
\hline$\leq 4$ & 1.00 & 0.116 & 1.00 & 0.142 \\
\hline$>4$ & $1.534(0.900-2.615)$ & & $1.572(0.859-2.875)$ & \\
\hline \multicolumn{5}{|c|}{ Symptom from brain metastases at brain recurrence } \\
\hline No & 1.00 & 0.376 & 1.00 & 0.499 \\
\hline Yes & $1.310(0.721-2.380)$ & & $1.233(0.672-2.260)$ & \\
\hline \multicolumn{5}{|c|}{ Extracranial disease at brain recurrence } \\
\hline Controlled & 1.00 & $<0.001$ & 1.00 & 0.055 \\
\hline Uncontrolled & $3.059(1.665-5.622)$ & & $1.855(0.987-3.600)$ & \\
\hline \multicolumn{5}{|c|}{ Chemotherapy after brain recurrence } \\
\hline No & 1.00 & $<0.001$ & 1.00 & 0.01 \\
\hline Yes & $0.174(0.094-0.324)$ & & $0.457(0.250-0.832)$ & \\
\hline \multicolumn{5}{|c|}{ WBI or G-knife for brain recurrence } \\
\hline No & 1.00 & 0.001 & 1.00 & 0.050 \\
\hline Yes & $0.367(0.205-0.656)$ & & $0.533(0.283-1.001)$ & \\
\hline \multicolumn{5}{|c|}{ Time from first brain irradiation to brain recurrence, mo } \\
\hline$<6$ & 1.00 & 0.153 & - & - \\
\hline$\geq 6$ & $0.679(0.400-1.154)$ & & - & \\
\hline
\end{tabular}


Table 4 Characteristics of the ten patients who lived more than 1 year after brain recurrence

\begin{tabular}{|c|c|c|c|c|c|c|c|c|c|c|}
\hline & Patient 1 & Patient 2 & Patient 3 & Patient 4 & Patient 5 & Patient 6 & Patient 7 & Patient 8 & Patient 9 & Patient 10 \\
\hline OSrec, mo. & 45.8 & 13.2 & 13.6 & 12.1 & 57.8 & 15.6 & 15.1 & 12.8 & 24.7 & 41.5 \\
\hline $\begin{array}{l}\text { Age at brain } \\
\text { recurrence, years }\end{array}$ & 62 & 61 & 71 & 63 & 57 & 64 & 69 & 63 & 64 & 65 \\
\hline $\begin{array}{l}\text { ECOG PS score at } \\
\text { brain recurrence }\end{array}$ & 0 & 0 & 1 & 1 & 0 & 1 & 1 & 1 & 0 & 1 \\
\hline Sex & Male & Female & Female & Female & Female & Female & Female & Male & Male & Female \\
\hline $\begin{array}{l}\text { Initial disease } \\
\text { stage (limited or } \\
\text { extensive) }\end{array}$ & Extensive & Extensive & Extensive & Extensive & Extensive & Extensive & Extensive & Extensive & Limited & Limited \\
\hline $\begin{array}{l}\text { Extrathoracic } \\
\text { organs involved at } \\
\text { initial diagnosis }\end{array}$ & $\begin{array}{l}\text { Brain, } \\
\text { adrenal }\end{array}$ & Bone & Liver & Thyroid & Brain & Brain & Brain & None ${ }^{a}$ & None & None \\
\hline $\begin{array}{l}\text { No. of brain } \\
\text { lesions at brain } \\
\text { recurrence }(1,2-4 \\
\text { or } \geq 5)\end{array}$ & 1 & $2-4$ & $2-4$ & $2-4$ & $\geq 5$ & 1 & $\geq 5$ & 1 & 1 & 1 \\
\hline $\begin{array}{l}\text { Symptoms from } \\
\text { brain lesions at } \\
\text { brain recurrence } \\
\text { (no or yes) }\end{array}$ & No & No & No & No & No & No & No & No & No & Yes \\
\hline $\begin{array}{l}\text { Extracranial } \\
\text { disease at brain } \\
\text { recurrence }\end{array}$ & Controlled & Uncontrolled & Controlled & Controlled & Controlled & Controlled & Controlled & Controlled & Controlled & Uncontrolled \\
\hline $\begin{array}{l}\text { Time from the } \\
\text { end of first brain } \\
\text { irradiation to brain } \\
\text { recurrence, mo. }\end{array}$ & 8.3 & 11.3 & 2.5 & 4.6 & 13.4 & 16.2 & 7.5 & 5.1 & 8.9 & 5.1 \\
\hline $\begin{array}{l}\text { Treatment } \\
\text { received for brain } \\
\text { lesion after brain } \\
\text { recurrence }\end{array}$ & SRS & SRS & SRS & SRS & $\begin{array}{l}\text { Temozolo- } \\
\text { mide }\end{array}$ & $\begin{array}{l}\text { SRS (after } \\
\text { surgery) }\end{array}$ & $\begin{array}{l}\text { Temozolo- } \\
\text { mide }\end{array}$ & SRS & SRS & SRS \\
\hline $\begin{array}{l}\text { Chemotherapy } \\
\text { after brain } \\
\text { recurrence (no or } \\
\text { yes) }\end{array}$ & Yes & Yes & Yes & No & Yes & No & Yes & No & No & Yes \\
\hline $\begin{array}{l}\text { Additional brain } \\
\text { failure after initial } \\
\text { brain recurrence } \\
\text { after WBI (no or } \\
\text { yes) }\end{array}$ & Yes & No & Yes & Yes & Yes & Yes & Yes & Yes & Yes & No \\
\hline $\begin{array}{l}\text { Salvage therapy } \\
\text { for additional } \\
\text { brain failure after } \\
\text { initial brain } \\
\text { recurrence after } \\
\text { WBI }\end{array}$ & Chemo & - & Chemo & $\begin{array}{l}\text { SRS, } \\
\text { repeat } \\
\text { WBI } \\
\text { afterwards }\end{array}$ & SRS & SRS & SRS & Observation & Observation & - \\
\hline
\end{tabular}

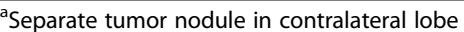

Abbreviations: OSrec overall survival time after brain recurrence, ECOG PS Eastern Cooperative Oncology Group performance status, SRS stereotactic radiosurgery, WBI whole-brain irradiation

significantly better survival than did patients with stable or progressive extracranial disease [13].

Our findings also showed that patients who received $\geq 4$ cycles of chemotherapy before brain recurrence had better survival, perhaps because of a lower systemic disease burden. We also found that patients who received chemotherapy after brain recurrence had better survival and better brain control than those who did not.
Although several new chemotherapy agents have been evaluated in phase II trials for second-line treatment of SCLC, to date they have not resulted in changes to standard practice, which is currently platinum plus etoposide for first-line therapy and topotecan for second-line therapy [18]. Further improvements in systemic therapy, such as targeted agents and immune checkpoint inhibitors, are eagerly awaited. 
We found that all 10 of the patients who lived for more than 1 year after brain recurrence received SRS, whether for initial or subsequent brain recurrence, a finding that highlights the importance of local treatment to improve outcome $[19,20]$.

The graded prognostic assessment (GPA) is advocated as a reliable and useful tool for estimating survival and for selecting appropriate treatment strategies for patients with brain metastases [21]. The original GPA score included age, KPS, number of brain lesions, and the presence or absence of extracranial metastases and has been validated for its prognostic potential in lung cancer $[22,23]$. Although we also identified PS and extracranial disease control as significant prognostic factors, we could not calculate GPA in our patient population because they not only had recurrent disease but they also tended to have a more extensive systemic disease burden than the patients included in the development of the GPA analysis.

This study had some limitations that should be considered in interpreting our results, chief among them being its retrospective nature, broad patient heterogeneity, and relatively small sample size. We cannot rule out the possibility that our findings could reflect selection bias. Thus our results should be considered exploratory and ideally should be validated in a larger, prospective cooperative group study.

\section{Conclusions}

The prognosis for patients with SCLC that recurs in the brain after WBI is still rather dismal, but survival in some cases, particularly those with adequate PS and controlled extracranial disease, can be extended if the recurrence is managed therapeutically.

\section{Abbreviations}

BCrec: Brain control after brain recurrence; ECOG: Eastern Cooperative Oncology Group; GPA: Graded prognostic assessment; KPS: Karnofsky performance score; OS: Overall survival; OSrec: Overall survival after brain recurrence;

PCI: Prophylactic cranial irradiation; PS: Performance status; SCLC: Small cell lung cancer; SRS: Stereotactic radiosurgery; WBI: Whole-brain irradiation

\section{Acknowledgments}

We appreciate the editorial contributions of Christine F. Wogan, MS, ELS, of MD Anderson's Division of Radiation Oncology, in the development and revision of this report.

\section{Funding}

This work was supported in part by the National Cancer Institute, National Institutes of Health [Cancer Center Support (Core) Grant P30 CA016672 to The University of Texas MD Anderson Cancer Center].

\section{Availability of data and materials}

Please contact author for data requests.

\section{Authors' contributions}

RS conceived the study, participated in its design and planning, oversaw the data collection and statistical analyses, and wrote the manuscript. XW and PKA extracted patient information from a departmental database, interpreted those data with regard to the nature and timing of the interventions and recurrence patterns, and conducted statistical analyses. JWW, JDC, RK, and SHL participated in the study design, provided patients for the analysis, oversaw their clinical care, and helped with the interpretation of the results. All authors read and approved the final manuscript.

\section{Ethics approval and consent to participate}

This retrospective study was approved by the Institutional Review Board of The University of Texas MD Anderson Cancer Center.

\section{Consent for publication}

Not applicable.

\section{Competing interests}

Dr. Welsh receives funding from The Family of M. Adnan Hamed; The Susan and Peter Goodwin Foundation; Mabuchi Research fund; the Weigand Foundation; and The Orr Family Foundation (to MD Anderson Cancer Center's Thoracic Radiation Oncology program). Dr. Lin has grants from Genentech, Hitachi Chemical Diagnostics, New River Labs, Peregrine, BeyondSpring Pharmaceuticals, and STCube Pharmaceuticals and is on the advisory board for AstraZeneca.

\section{Publisher's Note}

Springer Nature remains neutral with regard to jurisdictional claims in published maps and institutional affiliations.

Received: 10 April 2018 Accepted: 18 December 2018 Published online: 29 December 2018

\section{References}

1. Aupérin A, Arriagada R, Pignon JP, et al. Prophylactic cranial irradiation for patients with small-cell lung cancer in complete remission. Prophylactic cranial irradiation overview collaborative group. N Engl J Med. 1999;341(7): 476-784.

2. Bernhardt $D$, Adeberg $S$, Bozorgmehr $F$, et al. Outcome and prognostic factors in patients with brain metastases from small-cell lung cancer treated with whole brain radiotherapy. J Neuro-Oncol. 2017;134(1):205-12.

3. Nugent JL, Bunn PA Jr, Matthews MJ, et al. CNS metastases in small cell bronchogenic carcinoma: increasing frequency and changing pattern with lengthening survival. Cancer. 1979;44(5):1885-93.

4. Faivre-Finn C, Snee M, Ashcroft L, et al. CONVERT study team. Concurrent once-daily versus twice-daily chemoradiotherapy in patients with limitedstage small-cell lung cancer (CONVERT): an open-label, phase 3, randomised, superiority trial. Lancet Oncol. 2017;18(8):1116-25.

5. Turrisi AT 3rd, Kim K, Blum R, et al. Twice-daily compared with once-daily thoracic radiotherapy in limited small-cell lung cancer treated concurrently with cisplatin and etoposide. N Engl J Med. 1999;340(4):265-71.

6. Kushnirsky M, Nguyen V, Katz JS, et al. Time-delayed contrast-enhanced MRI improves detection of brain metastases and apparent treatment volumes. J Neurosurg. 2016;124(2):489-95.

7. Wong WW, Schild SE, Sawyer TE, Shaw EG. Analysis of outcome in patients reirradiated for brain metastases. Int J Radiat Oncol Biol Phys. 1996;34(3): 585-90.

8. Son $\mathrm{CH}$, Jimenez R, Niemierko A, Loeffler JS, Oh KS, Shih HA. Outcomes after whole brain reirradiation in patients with brain metastases. Int J Radiat Oncol Biol Phys. 2012;82(2):e167-72.

9. Scharp M, Hauswald H, Bischof M, Debus J, Combs SE. Re-irradiation in the treatment of patients with cerebral metastases of solid tumors: retrospective analysis. Radiat Oncol. 2014;3(9):4.

10. Aktan M, Koc M, Kanyilmaz G, Tezcan Y. Outcomes of reirradiation in the treatment of patients with multiple brain metastases of solid tumors: a retrospective analysis. Ann Transl Med. 2015;3(21):325.

11. Ozgen Z, Atasoy BM, Kefeli AU, Seker A, Dane F, Abacioglu U. The benefit of whole brain reirradiation in patients with multiple brain metastases Radiat Oncol. 2013;8:186.

12. Bernhardt D, Bozorgmehr F, Adeberg S, et al. Outcome in patients with small cell lung cancer re-irradiated for brain metastases after prior prophylactic cranial irradiation. Lung Cancer. 2016;101:76-81.

13. Harris $\mathrm{S}$, Chan MD, Lovato JF, et al. Gamma knife stereotactic radiosurgery as salvage therapy after failure of whole-brain radiotherapy in patients with small-cell lung cancer. Int J Radiat Oncol Biol Phys. 2012;83(1):e53-9. 
14. Sheehan J, Kondziolka D, Flickinger J, Lunsford LD. Radiosurgery for patients with recurrent small cell lung carcinoma metastatic to the brain: outcomes and prognostic factors. J Neurosurg. 2005;102(Suppl):247-54.

15. Caballero JA, Sneed PK, Lamborn KR, et al. Prognostic factors for survival in patients treated with stereotactic radiosurgery for recurrent brain metastases after prior whole brain radiotherapy. Int J Radiat Oncol Biol Phys. 2012;83(1):303-9.

16. Huang Z, Sun B, Shen G, et al. Brain metastasis reirradiation in patients with advanced breast cancer. J Radiat Res. 2017;58(1):142-8.

17. Maranzano E, Trippa F, Casale M, et al. Reirradiation of brain metastases with radiosurgery. Radiother Oncol. 2012;102(2):192-7.

18. Waqar SN, Morgensztern D. Treatment advances in small cell lung cancer (SCLC). Pharmacol Ther. 2017;180:16-23.

19. Gomez DR, Blumenschein GR Jr, Lee JJ, et al. Local consolidative therapy versus maintenance therapy or observation for patients with oligometastatic non-small-cell lung cancer without progression after firstline systemic therapy: a multicentre, randomised, controlled, phase 2 study. Lancet Oncol. 2016;17(12):1672-82.

20. Iyengar P, Wardak Z, Gerber DE, et al. Consolidative radiotherapy for limited metastatic non-small-cell lung cancer: a phase 2 randomized clinical trial. JAMA Oncol. 2018;4(1):e173501.

21. Sperduto PW, Berkey B, Gaspar LE, Mehta M, Curran W. A new prognostic index and comparison to three other indices for patients with brain metastases: an analysis of 1,960 patients in the RTOG database. Int J Radiat Oncol Biol Phys. 2008;70(2):510-4.

22. Sperduto PW, Chao ST, Sneed PK, et al. Diagnosis-specific prognostic factors, indexes, and treatment outcomes for patients with newly diagnosed brain metastases: a multi-institutional analysis of 4,259 patients. Int J Radiat Oncol Biol Phys. 2010;77(3):655-61.

23. Sperduto PW, Kased N, Roberge D, et al. Summary report on the graded prognostic assessment: an accurate and facile diagnosis-specific tool to estimate survival for patients with brain metastases. J Clin Oncol. 2012;30(4): 419-25.

Ready to submit your research? Choose BMC and benefit from:

- fast, convenient online submission

- thorough peer review by experienced researchers in your field

- rapid publication on acceptance

- support for research data, including large and complex data types

- gold Open Access which fosters wider collaboration and increased citations

- maximum visibility for your research: over $100 \mathrm{M}$ website views per year

At $\mathrm{BMC}$, research is always in progress.

Learn more biomedcentral.com/submissions 